\title{
Nuclear medium dependence of transverse Lambda polarisation in quasi-real photoproduction
}

\author{
Klaus Rith ${ }^{* \dagger}$ (on behalf of the HERMES collaboration) \\ Friedrich-Alexander Universität Erlangen-Nürnberg and DESY/Hamburg \\ E-mail: klaus.rith@desy.de
}

\begin{abstract}
Transverse polarization of $\Lambda$ hyperons produced inclusively in quasireal photon-nucleon scattering has been studied for several nuclear targets ranging from hydrogen to xenon. The transverse polarisation decreases with the atomic-mass number A of the nuclear target and vanishes for krypton and xenon. The spin transfer $D_{L L^{\prime}}^{\Lambda}$ from the longitudinally polarised lepton beam to a longitudinally polarised $\Lambda$ has been measured with improved accurary and is found to be positive and different from zero by nearly 5 standard deviations.
\end{abstract}

XVIII International Workshop on Deep-Inelastic Scattering and Related Subjects, DIS 2010 April 19-23, 2010

Firenze, Italy

\footnotetext{
* Speaker.

${ }^{\dagger}$ This work has been supported by the German BMBF (contract nr. 06 ER 9065 )
} 


\section{Introduction}

The $\Lambda$ hyperon is an especially interesting particle in spin physics. It is the lightest strange baryon $\left(M_{\Lambda}=1115.684 \mathrm{MeV}\right)$ and thus can only decay weakly $\left(\tau=2.632 \cdot 10^{-10} s\right)$, the dominant decay channels being $\Lambda \rightarrow p \pi^{-}(63.9 \%)$ and $\Lambda \rightarrow n \pi^{0}$ (35.8\%). In the HERMES experiment $\Lambda$ particles are studied via their charged decay channel. The weak decay is parity violating, leading to an angular distribution where the protons are preferentially emitted along the spin direction of their parent $\Lambda$. In the $\Lambda$ rest frame the angular distribution has the form

$$
\frac{d N}{d \Omega_{p}} \propto 1+\alpha P^{\Lambda} \cos \theta_{p}
$$

where $P^{\Lambda}$ is the magnitude of the $\Lambda$ polarisation, $\theta_{p}$ is the angle between the proton momentum and the direction of the $\Lambda$ polarisation and $\alpha=0.642 \pm 0.013$ is the analysing power of the parityviolating weak decay. The measurement of this angular distribution therefore offers the unique opportunity to study spin effects in the fragmentation process.

So far three aspects of $\Lambda$ polarisation have been studied in detail by the HERMES experiment:

- the longitudinal spin transfer $D_{L L^{\prime}}^{\Lambda}$ to the $\Lambda$ hyperon in semi-inclusive electron/positronnucleon scattering with a longitudinally polarised lepton beam [1];

- the longitudinal spin transfer $K_{L L^{\prime}}^{\Lambda}$ from a longitudinally polarised target to the $\Lambda$ hyperon [2];

- the transverse $\Lambda$ polarisation in quasireal photoproduction [3] and its dependence on the atomic-mass number $\mathrm{A}$ of the target.

In this contribution preliminary results for the nuclear-medium dependence of the transverse $\Lambda$ polarisation and for a more precise measurement of $D_{L L^{\prime}}^{\Lambda}$ are presented.

\section{Transverse $\Lambda$ polarisation}

Transverse polarisation $P_{n}^{\Lambda}$ of $\Lambda$ hyperons in inclusive production by high energy proton beams, $p N \rightarrow \Lambda X$, was first observed about 35 years ago. Since then this phenomenon has been studied in great detail by many experiments a large variety of hadron beams $\left(p, \bar{p}, n, \pi^{-}, K^{-}, K^{+}, \Sigma^{-}\right.$, heavy ions) and various target nuclei. For a review of early results and experiments see Ref. [4]. The general features of the polarisation measured in these experiments are: a) it is transverse to the production plane spanned by the momentum vectors of the beam and the produced $\Lambda$, the only direction allowed for unpolarised beams and targets by parity conservation in strong and electromagnetic interactions; b) $P_{n}^{\Lambda}$ is negative for most of the hadron beams but positive for $K^{-}$and $\Sigma^{-}$ beams; c) $P_{n}^{\Lambda}$ rises with $p_{T}$, the $\Lambda$ momentum transverse to the beam, for $p_{T}$ values up to about $1 \mathrm{GeV}$, then it is flat up to the highest measured $p_{T}$ values of about $3 \mathrm{GeV}$; d) at fixed $p_{T}$ the polarisation rises with the Feynman variable $x_{F}=p_{L}^{*} / p_{L, \text { max }}^{*}$, where $p_{L}^{*}$ is the component of the hyperon momentum in beam direction measured in the beam-target CMS and $p_{L, \text { max }}^{*}$ its maximal possible value; e) $P_{n}^{\Lambda}$ depends only slightly on the beam momentum and the atomic-mass number A of the target nuclei. No difference was observed between hydrogen $(\mathrm{H})$ and deuterium $(\mathrm{D})$ targets 
within the statistical accuracy of the measurements while the polarisation from a beryllium target appeared to be slightly smaller than that from $\mathrm{H}$ and $\mathrm{D}$ [5], and a reduction of the magnitude of the polarisation by about $20 \%$ was observed for $\mathrm{Cu}$ and $\mathrm{Pb}$ targets compared to $\mathrm{Be}$ [6].

Transverse $\Lambda$ polarisation has also been studied in inclusive electron/photon-nucleon interactions $e N \rightarrow \Lambda X$. While the results of previous experiments [7,8] were inconclusive due to their limited statistical accuracy, the HERMES experiment observed a substantial non-zero polarisation in quasireal photoproduction [3], $P_{n}^{\Lambda}$ being positive as for $K^{-}$and $\Sigma^{-}$beams. This might indicate that the $\gamma \rightarrow s \bar{s}$ hadronic component of the photon plays a significant role in inclusive quasireal photoproduction of $\Lambda$ particles. Below we present preliminary results for the nuclear-mass dependence of this polarisation.

\section{The HERMES spectrometer and data analysis}

The $\Lambda$ electroproduction data used in this analysis where accumulated in the years 1996-2005 by the HERMES experiment at the HERA e-p accelerator facility with a $27.6 \mathrm{GeV}$ electron/positron beam and various nuclear gas targets $\left({ }^{1} \mathrm{H},{ }^{2} \mathrm{H},{ }^{3} \mathrm{He},{ }^{4} \mathrm{He}\right.$, and the heavier gases $\mathrm{N}, \mathrm{Ne}, \mathrm{Kr}$ and $\mathrm{Xe}$ in natural abundance) internal to the lepton storage ring. The HERMES detector is described in detail in Ref. [9]. The criteria for data selection and the analysis procedure are identical to those described in Ref. [3]. The $\Lambda$ events were detected by requiring the presence of at least two hadron candidates of opposite charge. It was assured with the help of a $\breve{C}$ erenkov detector that the positive hadron was not a pion. Two spatial vertices where reconstructed for each event, i.e., the decay vertex from the intersection of the proton and pion tracks and the $\Lambda$-production vertex from the intersection of the reconstructed $\Lambda$ track with the nominal beam axis. The distance between the two vertices was required to be larger than $15 \mathrm{~cm}$. Additional vertex and fiducial-volume cuts were used to suppress combinatorial background. The scattered positron was not requested for this analysis and therefore the data sample of this inclusive measurement, $e N \rightarrow \Lambda X$, is dominated by events from quasireal photoproduction. The invariant-mass distribution was fitted with a Gaussian plus a second order polynomial. Events within a window of $\pm 3.3 \sigma$ around the mean value of the Gaussian fit were selected for further analysis. The extraction of the $\Lambda$ polarization from the data was accomplished using the moment method which exploits the top/bottom symmetry of the detector [3]. The systematic uncertainty of the polarisation measurement was estimated using pairs of oppositely charged hadrons that originated from the target region within two mass windows above and below the $\Lambda$ mass window. In addition the false asymmetry measured for charged pion pairs from the decay $K_{s}^{0} \rightarrow \pi^{+} \pi^{-}$has been determined.

\section{Results for $P_{n}^{\Lambda}$}

The results for $P_{n}^{\Lambda}$ published in Ref. [3] were based on approximately $260 \cdot 10^{3} \Lambda$ events collected in the years 1996-2000 using mostly hydrogen and deuterium targets. The data for all targets were combined and an average $\Lambda$ polarisation of $P_{n}^{\Lambda}=0.078 \pm 0.006$ (stat) \pm 0.012 (syst) was obtained. The main features of the data are presented in Ref. [3]. Much more data were collected in the years 2002-2005 resulting in approximately $385 \cdot 10^{3} \Lambda$ events. The increased statistics collected especially with hydrogen and deuterium and the heavy target nuclei $\mathrm{Kr}$ and $\mathrm{Xe}$ allowed to 

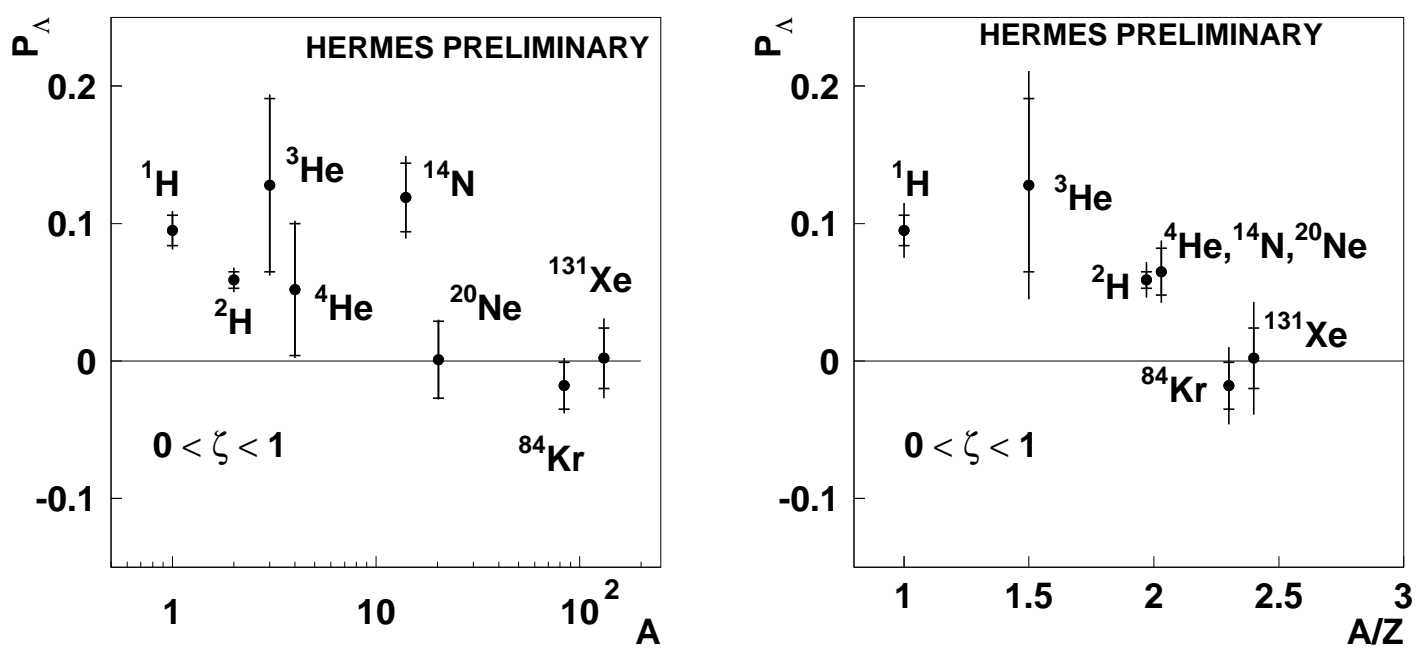

Figure 1: Transverse $\Lambda$ polarization versus atomic-mass number of the various target nuclei (left) and versus $\mathrm{A} / \mathrm{Z}$ (right).

study the dependence of the transverse $\Lambda$ polarisation on the atomic-mass number A of the target nuclei. Preliminary results for $P_{n}^{\Lambda}$ integrated over the experimental kinematics are presented versus $A$ in the left panel of Fig. 1 and versus the ratio A/Z in the right panel of the figure. A clear indication of an A-dependence of $P_{n}^{\Lambda}$ is observed: the polarization for the light nuclei $\left({ }^{1} \mathrm{H},{ }^{2} \mathrm{H}\right)$ is statistically significant positive, while it is compatible with zero within the statistical uncertainty for the heavier nuclei $\mathrm{Kr}$ and $\mathrm{Xe}$. There is also an indication that the polarisation obtained from the hydrogen target is larger than that for the deuterium target. They differ by about 2.1 standard deviations of the total uncertainty. The origin of this nuclear medium effect is presently not understood.

\section{New results for the longitudinal spin transfer $D_{L L^{\prime}}^{\Lambda}$}

In experiments with longitudinally polarised electron/positron beams the beam polarisation can be transferred to the produced $\Lambda$ hyperon. The component of the polarisation transferred along the direction L' from the exchanged virtual photon to the produced $\Lambda$ is given by

$$
P_{L^{\prime}}^{\Lambda}=P_{b} D(y) D_{L L^{\prime}}^{\Lambda}
$$

where $\mathrm{L}$ is the primary quantization axis, directed along the virtual-photon momentum, $P_{b}$ is the beam polarisation, $y=v / E$ is the ratio of the energies of the virtual photon and the incident lepton and $\mathrm{D}(\mathrm{y})$ is the depolarisation factor taking into account the loss of polarisation of the virtual photon as compared to that of the incident lepton. The longitudinal spin-transfer coefficient $D_{L L^{\prime}}^{\Lambda}$ contains information about the spin transfer in the fragmentation of the struck quark and/or the target remnant in the fragmentation process. In contrast to the measurements of $P_{n}^{\Lambda}$ the scattered electron has been detected in the semi-inclusive measurements of the longitudinal $\Lambda$ polarisation, $e N \rightarrow e^{\prime} \Lambda X$, allowing the determination of the full kinematics. Results for $D_{L L^{\prime}}^{\Lambda}$ based on the 


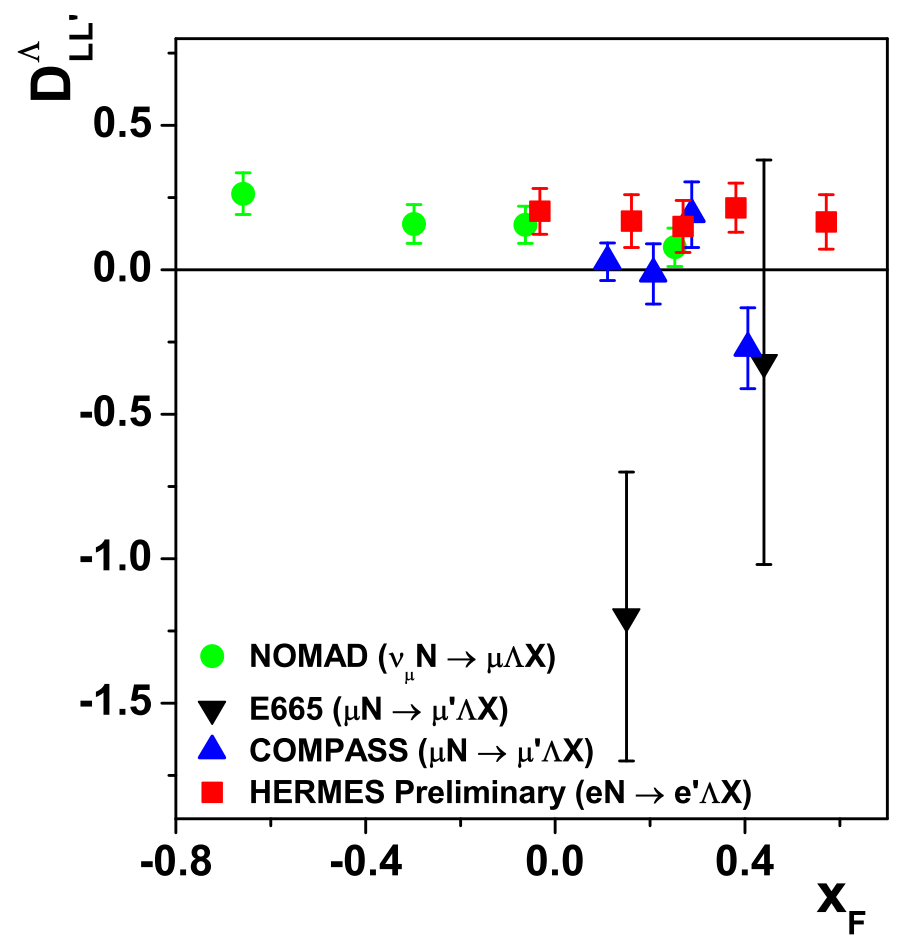

Figure 2: Preliminary HERMES results for the longitudinal spin transfer $D_{L L^{\prime}}^{\Lambda}$ versus $x_{F}$ together with results from NOMAD [10], E665 [11] and COMPASS [12].

data taken in the years 1996-2000 have been presented in Ref. [1]. The spin transfer to the $\Lambda$ along its momentum direction, averaged over the kinematic region with $Q^{2}>0.8 \mathrm{GeV}^{2}, y<0.85$, and $x_{F}>0$ extracted from these data had the value $D_{L L^{\prime}}^{\Lambda}=0.11 \pm 0.10$ (stat) \pm 0.03 (syst). Here $-Q^{2}$ is the squared four-momentum of the virtual photon. The substantially increased statistics collected in the years 2002-2007 allowed a much more precise determination of $D_{L L^{\prime}}^{\Lambda}$ and detailed studies concerning the choice of the secondary quantisation axis, resulting in the conclusion that the correct choice is the direction of the virtual photon. The preliminary results are shown in Fig. 2 as a function of $x_{F}$ together with data obtained by the NOMAD experiment [10] at CERN with a $43 \mathrm{GeV} v_{\mu}$-beam, by the Fermilab E665 experiment [11] with a polarized $470 \mathrm{GeV}$ muon beam and the COMPASS experiment [12] at CERN with a $160 \mathrm{GeV}$ polarised muon beam. The preliminary result averaged over the experimental kinematics is $D_{L L^{\prime}}^{\Lambda}=0.19 \pm 0.04$, and therefore different from zero by nearly 5 standard deviations in contrast to the recent COMPASS result which is compatible with zero. Surprisingly the HERMES results at positive $x_{F}$ are in good agreement with the NOMAD results at negative $x_{F}$.

\section{References}

[1] HERMES Collaboration, A. Airapetian et al., Phys. Rev. D 74, 072004 (2006).

[2] D. Veretennikov, Proceedings of the 16th International Workshop on Deep Inelastic Scattering and Related Subjects (DIS 2008), London, UK (04/07/2008-04/11/2008), Sciencewise Publishing, Berlin, 2008, doi:10.3360/dis.2008.212. 
[3] HERMES Collaboration, A. Airapetian et al., Phys. Rev. D 76, 092008 (2007).

[4] A.D. Panagiotou, Int. J. Mod. Phys. A5, 1197 (1990).

[5] K. Raychaudhuri et al., Phys. Lett. 90B, 319 (1980).

[6] L. Pondrom, Phys. Rep. 122, 57 (1985).

[7] SLAC-BC-072 Collaboration, K. Abe et al., Phys. Rev. D 29, 1877 (1984).

[8] CERN-WA-004 Collaboration, D. Aston al., Nucl. Phys. B195, 189 (1982).

[9] HERMES Collaboration, K. Ackerstaff et al., Nucl. Instrum. Methods A417, 230 (1998).

[10] NOMAD Collaboration, P. Astier et al., Nucl. Phys. B588, 3 (2000).

[11] E665 Collaboration, M.R. Adams et al., Eur. Phys. J. C 17, 263 (2000).

[12] COMPASS Collaboration, M. Alekseev et al., Eur. Phys. J. C 64, 171 (2009). 